\title{
émulations
}

\section{Yannick Duvauchelle - Les représentations de la réalité. Étude sociologique}

\section{Jean Frances}

Émulations - Revue de sciences sociales

2016, «Comptes rendus critiques, En ligne »

\section{Article disponible à l'adresse suivante}

https://ojs.uclouvain.be/index.php/emulations/article/view/7153

\section{Pour citer cet article}

Jean Frances, «Yannick Duvauchelle — Les représentations de la réalité. Étude sociologique », Émulations, en ligne. Mise en ligne le 24 mai 2016.

DOI : 10.14428/emulations.cr.019

Distribution électronique : Université catholique de Louvain (Belgique) : ojs.uclouvain.be

(C) Cet article est mis à disposition selon les termes de la Licence Creative Commons Attribution, Pas d'Utilisation Commerciale 4.0 International. http://creativecommons.org/licenses/by-nc/4.0/

Éditeur : Émulations - Revue de sciences sociales / Presses universitaires de Louvain https://ojs.uclouvain.be/index.php/emulations

ISSN électronique : 1784-5734

UCL PRESSES

UNIVERSITAIRES 


\section{Yannick Duvauchelle - Les représenta- tions de la réalité. Étude sociologique}

Jean Frances ${ }^{1}$

Recensé : Yannick Duvauchelle, Les représentations de la réalité. Étude sociologique, Aix-en-Provence, Presses universitaires de Provence, 2016.

S’intéressant aux manières dont les individus génèrent des "propositions », Yannick Duvauchelle entend « observer de quelle manière et selon quels principes les acteurs sociaux se représentent la réalité » (p.11). Pour l'auteur, les "propositions » sont des « dits et des écrits » ayant pour objectif « de témoigner d'une réalité » (Ibid.). L’ouvrage s'inscrit « dans le cadre d'une sociologie de la connaissance [...] [qui] incite[rait] à regarder les connaissances sous le prisme de données sociales » (p. 15). Au long des six courts chapitres de ce livre extrait d'un travail de thèse, Y. Duvauchelle cherche à « examiner s'il n'est pas possible de trouver », dans les «données sociales », «le principe de détermination » des connaissances (Ibid.). Dit autrement, l'auteur semble vouloir définir en quoi les possibilités de produire un énoncé relatif à une réalité sont socialement déterminées.

Pour essayer de mettre au jour les manières et les principes au fondement des capacités des acteurs à se représenter la réalité, l'auteur a opéré de manière inductive «dans une première phase exploratoire d'abord guidée par la curiosité [...] l'on s'est laissé libre d'approcher quelque proposition que ce soit (concrètement n'importe quel livre ou article)» (p. 20). Estimant nécessaire d'engager « une phase de systématisation des interrogations », Y. Duvauchelle a resserré ses terrains. Il étudie premièrement des «propositions scientifiques», puis des "propositions journalistiques» et enfin des «propositions militantes ». Il veut déterminer ce qui distingue et rapproche ces différentes modalités de « mise en mots » de la réalité. Pour ce faire, il a mené des entretiens avec six chercheurs (un professeur d'université en cinéma, une chercheuse en psychologie cognitive, trois doctorants dont deux en psychologie cognitive et un en musicologie et un étudiant en master 2), trois journalistes et cinq militants (deux « autour de la cause LGBT », un syndicaliste étudiant, un directeur d'un festival de cinéma et un directeur de cinéma). Les verbatim de ces interviews constituent le principal matériau d'enquête mobilisé, mais Y. Duvauchelle complète ces données avec des extraits de journaux et d'émissions télévisés (Journal télévisé de TF1, l'émission de critique ciné-

${ }^{1}$ Docteur en sociologie, chercheur associé au GSPR/EHESS. 
matographique Le Cercle, diffusée sur la chaîne Canal+). Il s'agit ainsi de « faire se rencontrer, sous le coup d'un questionnement unique, des matériaux et sources multiples » (p. 22) et d'ancrer empiriquement son analyse. Il s'agit aussi de postuler « qu'il n'est rien dans la réalité qui corresponde à des faits et suggérer que la reconnaissance perpétuelle de telles entités par les acteurs indique plutôt l'implication d'un ressort propositionnel spécial : l'observation que la façon dont les acteurs pensent la réalité passe ponctuellement par la reconnaissance de faits ne doit pas constituer l'emblème d'une espèce de succès méthodologique quant à la divulgation d'un aspect de la réalité premier ou fondamental, mais signaler plutôt la contribution privilégiée dans la constitution des propositions en question, de concepts et recours représentationnels dont le niveau élevé d'institution explique que - relativement à la considération de tel ou tel phénomène - leur usage apparaisse comme naturel, voire impératif, comme découler des phénomènes eux-mêmes » (p. 45).

Si l'auteur insiste sur l'ancrage empirique de son enquête c'est parce qu'à ses yeux, «les travaux se revendiquant de la sociologie de la connaissance demeurent trop en retrait ou trop à distance des connaissances interrogées, au sens où ils ne témoignent pas suffisamment étroitement du phénomène propositionnel » (p. 17). D'ailleurs, continue-t-il, ce même courant « laisse [...] trop "impensée" la question des connaissances en tant que telles, c'est-à-dire la question de leur constitution et des manières de faire dont elles sont le fait » (p. 19). C’est une position osée, pour ne pas dire plus. Cette question est «pensée » depuis longtemps. Et elle est même analysée sociologiquement. Il n'est qu'à mentionner les travaux d'A. Schütz ou ceux de P. Berger et T. Luckmann. D’ailleurs, il serait trop long ici de faire la liste des sociologues continuant d'investir cet objet. Les modalités d'articulation entre «manières de faire » incorporées par les acteurs et capacités à dire et écrire leur monde sont, elles aussi, examinées depuis longtemps : N. Elias ou P. Bourdieu, pour ne citer qu'eux, se sont attelés à la question. Si la revue de littérature paraît succincte, il n'en reste pas moins que l'ambition affichée par l'auteur éveille la curiosité du lecteur.

Le positionnement théorique de l'auteur est difficilement perceptible. En effet, l'écriture de Y. Duvauchelle est compliquée et les discussions relatives aux travaux de sociologie de la connaissance sont rares. Toutefois, l'auteur distingue trois plans ontologiques : la réalité, les mots et les concepts. Les mots utilisés par les acteurs ne sont pas la réalité mais renvoient à des concepts. Ces concepts renverraient à leur tour aux noyaux substantiels des réalités observées et vécues par les individus. Ainsi, faire des propositions, c'est user de mots qui désignent des concepts, lesquels référencent la réalité..., sans totalement la saisir : comme « les mots ne rapportent jamais à autre chose qu'à des concepts, on comprend que sous l'apparence de dire la vérité, sous l'apparence d'établir le vrai, il y a là l'avancement d'une certaine conception, il n'y a là que l'expression d'un certain façonnement conceptuel » (p. 59). Là encore, aucune mention n'est faite aux différentes théories du langage et on ne sait trop d'où l'auteur tient les distinctions conceptuelles qu'il propose. Il revendique ensuite un certain relativisme. D’après 
lui, «il n'est pas de moyen de décrire absolument la réalité, et donc pas non plus de moyen pour affirmer qu'une proposition est plus vraie qu'une autre » (p. 67). D'ailleurs, affirme-t-il plus haut dans son texte, « Ce à quoi doit se livrer la sociologie [sic.], c'est non pas rechercher les conditions de découverte de la vérité vraie, mais démonter la mécanique générale du savoir et des connaissances, rendre compte des conditions faisant qu'un matériel représentationnel acquière au sein de l'espace social le statut de vérité» (p. 65).

Venons-en à la thèse centrale de l'ouvrage. Pour Y. Duvauchelle, les modes de construction des propositions scientifiques ne se distinguent pas fondamentalement des modes journalistiques ou militants. Il n'y a pas entre eux de différences profondes. Ici, les références manquent encore et le positionnement n'est à nouveau pas très clair. Néanmoins, affirme-t-il, les modes scientifiques se différencient en ce que les chercheurs, prendraient explicitement en charge "le contenu conceptuel des mots employés » et qu’ils veilleraient à « délimiter le signalement conceptuel [...] [de leurs] mots » (p. 86). Puis, continue-t-il, tous les phénomènes physiques ou sociaux sont plus complexes que ce que les mots peuvent en dire : les phénomènes ne se laissent pas enserrer dans le langage. Dès lors, parler de la réalité, à savoir, faire des propositions c'est bien user d'abstractions artificiellement délimitées : cela vaut pour tous, militants chercheurs, journalistes, enfants... Faire des propositions en scientifiques, ça serait user d'abstractions en explicitant leurs modalités de délimitation et de conjugaisons avec d'autres abstractions. Et, par rapport aux quidams, les chercheurs auraient une compétence supplémentaire ou, du moins, plus affutée, celle de produire des concepts. Ainsi, leur travail participerait d'une « inflation conceptuelle faisant que [...] le regard posé sur la réalité n'est plus le même » (p.101). Voilà ce qui rapproche et distingue les façons scientifiques, journalistiques et militantes de se représenter et de dire la réalité. Il n'est pas trop de dire que la thèse manque quelque-peu d'originalité. 